\title{
Non-Fermi-liquid scattering rates and anomalous band dispersion in ferropnictides
}

\author{
J. Fink, ${ }^{1}$ A. Charnukha, ${ }^{1,}{ }^{*}$ E. D. L. Rienks, ${ }^{2}$ Z. H. Liu, ${ }^{1}$ S. Thirupathaiah, ${ }^{2, \dagger}$ I. Avigo, ${ }^{3}$ F. Roth, ${ }^{4}$ H. S. Jeevan,${ }^{5, \dagger}$ P. Gegenwart, ${ }^{5}$ \\ M. Roslova, ${ }^{1,6}$ I. Morozov, ${ }^{1,6}$ S. Wurmehl, ${ }^{1,7}$ U. Bovensiepen, ${ }^{3}$ S. Borisenko, ${ }^{1}$ M. Vojta, ${ }^{8}$ and B. Büchner ${ }^{1,7}$ \\ ${ }^{1}$ Leibniz Institute for Solid State and Materials Research Dresden, Helmholtzstrasse 20, D-01069 Dresden, Germany \\ ${ }^{2}$ Helmholtz-Zentrum Berlin, Albert-Einstein-Strasse 15, D-12489 Berlin, Germany \\ ${ }^{3}$ Fakultät für Physik, Universität Duisburg-Essen, Lotharstrasse 1, D-47075 Duisburg, Germany \\ ${ }^{4}$ Center for Free-Electron Laser Science/DESY, Notkestrasse 85, D-22607 Hamburg, Germany \\ ${ }^{5}$ Institut für Physik, Universität Augsburg, Universitätstrasse 1, D-86135 Augsburg, Germany \\ ${ }^{6}$ Department of Chemistry, Lomonosov Moscow State University, 119991 Moscow, Russia \\ ${ }^{7}$ Institut für Festkörperphysik, Technische Universität Dresden, D-01062 Dresden, Germany \\ ${ }^{8}$ Institut für Theoretische Physik, Technische Universität Dresden, D-01062 Dresden, Germany \\ (Received 9 March 2015; revised manuscript received 9 July 2015; published 12 November 2015)
}

\begin{abstract}
Angle-resolved photoemission spectroscopy is used to study the band dispersion and the quasiparticle scattering rates in two ferropnictide systems. We find the scattering rate for any given band to depend linearly on energy but to be independent of the control parameter. We demonstrate that the linear energy dependence gives rise to a weakly dispersing band with a strong mass enhancement when the band maximum crosses the chemical potential. The resulting small effective Fermi energy favors a BCS [J. Bardeen et al., Phys. Rev. 108, 1175 (1957)] -Bose-Einstein [S. N. Bose, Z. Phys. 26, 178 (1924)] crossover state in the superconducting phase.
\end{abstract}

DOI: 10.1103/PhysRevB.92.201106

Introduction. Unconventional/high-temperature superconductivity is observed in heavy fermion systems, cuprates, molecular crystals, and ferropnictides close to a point in the phase diagram where, as a function of a control parameter such as pressure, chemical pressure, or doping, antiferromagnetic order is suppressed [1,2]. A widespread view is that at this point, which is called a quantum critical point (QCP), strong antiferromagnetic fluctuations are a candidate for the glue mediating superconductivity and that these fluctuations would also account for the normal state non-Fermi-liquid behavior which is expressed, e.g., in a linear temperature dependence of the resistivity or in a large mass enhancement of the charge carriers. In the ferropnictides [3] the strange normal state properties have been observed, e.g., in transport [4] and thermal properties [5]. Theoretically a QCP in ferropnictides has been predicted in Refs. [6,7]. On the other hand, there are various models to explain the strange normal state properties of unconventional superconductors, e.g., for cuprates on the basis of a van Hove scenario [8-10].

Angle-resolved photoemission spectroscopy (ARPES) is a versatile method to obtain information on the energy $(E)$ and momentum (k) dependent real and imaginary parts of the self-energy function $\Sigma(E, \mathbf{k})$ which are related to the mass enhancement and the scattering rate, respectively [11-13].

In this contribution we used ARPES to study the band dispersion and the scattering rate $S(E)$ as a function of the control parameter of two ferropnictide [3] systems: the "122" compounds $\mathrm{BaFe}_{2}\left(\mathrm{As}_{1-x} \mathrm{P}_{x}\right)_{2}$ and $\mathrm{EuFe}_{2}\left(\mathrm{As}_{1-x} \mathrm{P}_{x}\right)_{2}$, in which As is isovalently substituted by $\mathrm{P}$ leading to chemical pressure

\footnotetext{
*Present address: Department of Physics, University of California, San Diego, La Jolla, California 92093, USA.

${ }^{\dagger}$ Present address: Solid State and Structural Chemistry Unit, Indian Institute of Science, Banglore-560012, India.

${ }^{\ddagger}$ Present address: Department of Physics, PESITM, Sagar Road, 577204 Shimoga, India.
}

PACS number(s): 74.25.Jb, 74.62.Dh, 74.72.Ek, 79.60.-i and the electron doped " 111 " compounds $\mathrm{NaFe}_{1-x} \mathrm{Co}_{x}$ As and $\mathrm{NaFe}_{1-x} \mathrm{Rh}_{x}$ As. There are numerous ARPES studies on these systems published in the literature [14-21]. In the present study we have measured along three directions in the Brillouin zone (BZ), shown in Fig. 1(a), to reach the high symmetry points on the three hole pockets and on the electron pockets.

In the wide range of the investigated control parameter we find linear-in-energy scattering rates independent of the control parameter and no enhancement at the supposed QCP. Furthermore, we see that at optimal "doping" we observe a crossing of the top of the hole band through the Fermi level. On the basis of these ARPES results we propose the following scenario: a co-action between a highly correlated electron liquid with a linear-in-energy scattering rate and a crossing of the top (bottom) of a hole (electron) pocket through the chemical potential causes an anomalous band dispersion at the Fermi level which leads to a strong mass enhancement in the normal state and to a small effective Fermi energy favoring a BCS [23] -Bose-Einstein (BE) [24] crossover state in the superconducting phase.

Experiment. Single crystals were grown using the self-flux technique $[25,26]$. ARPES measurements were conducted at the $1^{2}$ - and $1^{3}$-ARPES endstations attached to the beamline UE112 PGM 2 at BESSY with energy and angle resolutions between 4 and $10 \mathrm{meV}$ and $0.2^{\circ}$, respectively. Variable photon energies $h v=20-130 \mathrm{eV}$ were used to reach different $k_{z}$ values in the BZ. If not otherwise stated the measuring temperature at the $1^{2}$ - and $1^{3}$-ARPES endstations were 30 and $0.9 \mathrm{~K}$, respectively. Temperatures above the Néel temperature were used for unsubstituted samples in order to keep the samples in the paramagnetic state.

Although ARPES is a highly surface sensitive method, previous experimental and theoretical studies using density functional theory (DFT) band structure calculations show that ARPES results are close to the bulk electronic structure. In the 122 compounds a disordered $\mathrm{Ba} / \mathrm{Eu}$ surface layer is formed upon cleaving at low temperatures which leads to additional 

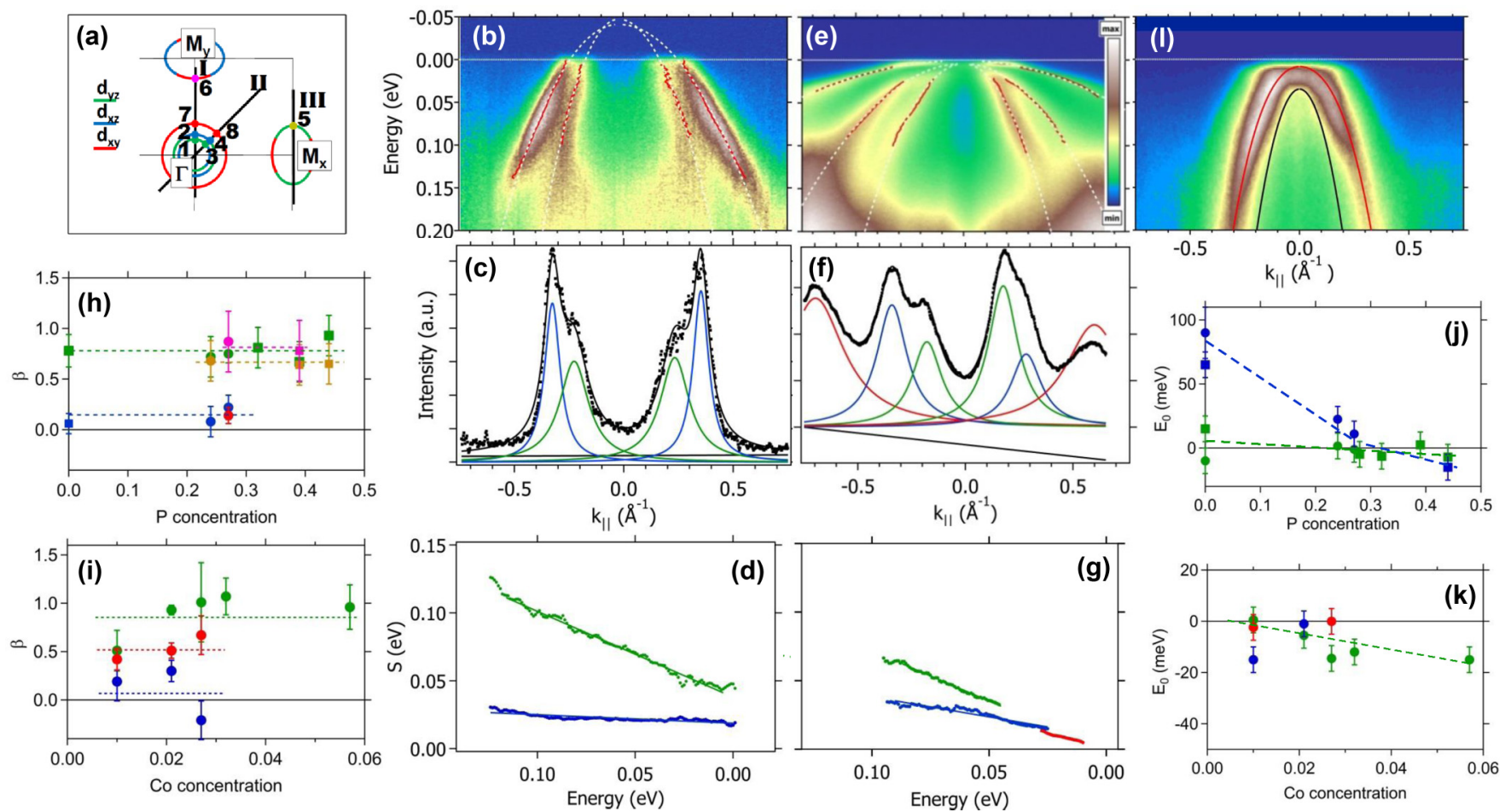

FIG. 1. (Color online) (a) Schematic Fermi surfaces of ferropnictides at $k_{z}=0$ formed by three hole pockets at $\Gamma$ and electron pockets at $M$. The back-folded electron pockets are not shown, since the ARPES matrix elements are rather small. The orbital character which was adopted from Ref. [22] is indicated by different colors. The color code of the high symmetry points is used in the panels (c), (d), (f), (g), and (h)-(k). (b) ARPES EDM of $\mathrm{BaFe}_{2}\left(\mathrm{As}_{1-x} \mathrm{P}_{x}\right)_{2}, x=0.27$ measured along cut I near $Z$ with a photon energy $h v=62 \mathrm{eV}$. The red lines depict dispersions derived from MDC fits. The dashed white line is a parabolic fit to these data. (c) MDC from the data in (b) at the binding energy $E_{B}=40 \mathrm{meV}$. (d) Scattering rate $S(E)$ (dots) together with a linear fit (solid line). (e)-(g) Analogous data for $\mathrm{NaFe}_{1-x} \mathrm{Rh}_{x} \mathrm{As}, x=0.027$ near $\Gamma$ measured with $h v=21 \mathrm{eV}$. (h) and (i) Doping-dependent $\beta$ values for various high symmetry points near the $\Gamma$ point of Ba/Eu122 (Ba dots/Eu squares) and $\mathrm{NaFe}_{1-x} \mathrm{Co}_{x} \mathrm{As}$ as a function of the P/Co concentration, respectively. (j) and (k) Doping-dependent top of the hole pockets near the $\Gamma$ point in $\mathrm{Ba} / \mathrm{Eu} 122$ and $\mathrm{NaFe}_{1-x} \mathrm{Co}_{x} \mathrm{As}$, respectively. (1) EDM of the inner hole pocket of $\mathrm{BaFe}_{2}\left(\mathrm{As}_{1-x} \mathrm{P}_{x}\right)_{2}, x=0.24$ near $\Gamma$ measured with $h v=80 \mathrm{eV}$ together with a marginal Fermi-liquid fit (red line) using the bare particle dispersion shown by a black line. The dashed lines in (h)-(k) are guides to the eye.

broadening of the lines due to elastic scattering [27]. Ordering at higher temperatures leads to backfolding of bands. In the 111 systems calculations indicate that the electronic structure of the surface layer is close to that of the bulk [28].

Results. First, we present data of the scattering rates. In Fig. 1(b) we show an energy distribution map (EDM) along cut I [Fig. 1(a)] for the optimally "doped" $\mathrm{BaFe}_{2}\left(\mathrm{As}_{1-x} \mathrm{P}_{x}\right)_{2}$, $x=0.27$ (with a superconducting transition temperature $T_{c}=$ $30 \mathrm{~K})$ measured close to the $Z=(0,0, \pi / c)$ point, where $c$ is the lattice constant. We first present data at this point of the BZ since at optimal doping only in this case finite Fermi velocities are observed which allow the evaluation of $S(E)$ down to low energies, while near $\Gamma$ the Fermi velocities are close to zero leading to less accurate data of $S(E)$ (see below). The two inner hole pockets can be clearly resolved. Due to matrix element effects the outer hole pocket is more difficult to see in this system. A fit to four Lorentzians is shown in Fig. 1(c). In Fig. 1(d) we present $S(E)$ calculated from the width in momentum space [13]. For both hole pockets, we find a non-Fermi-liquid linear-in-energy dependence in a large energy range of $5 \leqslant E \leqslant 120 \mathrm{meV}$, which can be described by $S(E)=\alpha+\beta E$. The value of $\alpha$ is determined by elastic scattering, while $\beta$ is governed by inelastic scattering processes. $\beta$ is much larger for the inner hole pocket compared to the value of the middle hole pocket. Similar results have been obtained previously for the system $\mathrm{Ba}\left(\mathrm{Fe}_{1-x} \mathrm{Co}_{x}\right)_{2} \mathrm{As}_{2}[29,30]$. In Fig. 1(h) we present a compilation of all available data of $\beta$ for $\mathrm{BaFe}_{2}\left(\mathrm{As}_{1-x} \mathrm{P}_{x}\right)_{2}$ and $\mathrm{EuFe}_{2}\left(\mathrm{As}_{1-x} \mathrm{P}_{x}\right)_{2}$ taken with photon energies corresponding to the $\Gamma$ point. No enhancement near optimal doping $(x=0.3)$ is detected.

In Fig. 1(e) we show an EDM of the optimally doped $\mathrm{NaFe}_{1-x} \mathrm{Rh}_{x}$ As $x=0.027\left(T_{c}=20 \mathrm{~K}\right)$ close to the $\Gamma$ point along cut I. All three hole pockets can be clearly resolved. The data at constant $E_{B}=40 \mathrm{meV}$ could be well fitted by six Lorentzians as shown in Fig. 1(f). The derived scattering rates $S$ are presented in Fig. 1(g). The 111 systems have the disadvantage that close to the Fermi level the middle hole pocket hybridizes with the inner hole pocket. As a consequence, reliable results on $S$ can be only derived at higher binding energies. For all three hole pockets, $S$ increases linearly with increasing energy in the range where there is no band overlap. For the inner, middle, and outer hole pockets we derive $\beta$ values of $1.0, \approx 0$, and 0.69 , respectively. In Fig. 1(i) we present a compilation of all $\beta$ values for $\mathrm{NaFe}_{1-x} \mathrm{Co}_{x} \mathrm{As}$ near $\Gamma$ as a function of Co concentration. As in the case of the 122 compounds, the $\beta$ values are rather independent of 
the control parameter and no enhancement at optimal doping $(x=0.027)$ is observed.

Second, we present data on the position of the hole pockets relative to the Fermi level. In Fig. 1(j) we show the top of the middle and the inner hole pocket in $\mathrm{BaFe}_{2}\left(\mathrm{As}_{1-x} \mathrm{P}_{x}\right)_{2}$ as derived from a parabolic fit to the measured dispersion. In the unsubstituted samples a splitting between $x z$ and the $y z$ states of the order of $50 \mathrm{meV}$ is observed in the tetragonal paramagnetic phase which is not expected since these bands should be degenerate at the $\Gamma$ point. On the other hand, a similar splitting has been observed $[31,32]$ above the structural transition temperature in ARPES experiments on detwinned crystals. This splitting was explained in terms of nematic fluctuations which exist above the Néel and the structural transition. As expected from other studies [32] the splitting decreases and disappears near optimal "doping." While we observe a decrease of the Fermi wave vector at $\Gamma$ with increasing "doping" concentration, at $Z$ the Fermi wave vector increases. Thus there is a Lifshitz transition of a Fermi cylinder at low $\mathrm{P}$ concentrations to an ellipsoid around the $Z$ point at higher $P$ concentrations. This Lifshitz transition close to optimal "doping" has been detected in in several ARPES studies of Ba/Eu122 systems "doped" with Co or P $[17,33,34]$. The proximity of the maximum of the inner hole pocket to the Fermi level near optimal doping is also supported by the EDM of $\mathrm{BaFe}_{2}\left(\mathrm{As}_{1-x} \mathrm{P}_{x}\right)_{2}$ shown in Fig. 1(1). We remark that in the present ARPES study, within error bars, we have not found any major differences in the electronic structure between $\mathrm{BaFe}_{2}\left(\mathrm{As}_{1-x} \mathrm{P}_{x}\right)_{2}$ and $\mathrm{EuFe}_{2}\left(\mathrm{As}_{1-x} \mathrm{P}_{x}\right)_{2}$. This is in line with the experimental observation that the Eu $4 f$ local magnetic moments only influence the phase diagram at low temperatures. Indeed, the superconducting transition is suppressed beyond $x=0.23$ due to the ferromagnetic ordering of Eu moments [35].

Finally, in Fig. 1(k) the top of the hole pockets in $\mathrm{NaFe}_{1-x} \mathrm{Co}_{x}$ As as a function of the Co concentration is depicted. As in the case of the 122 compounds, the crossing of the top of the inner hole pocket, having the strongest scattering rate, occurs near optimal doping. The proximity of the maximum of the hole pockets to the Fermi level near optimal doping is also supported by the EDM of $\mathrm{NaFe}_{1-x} \mathrm{Rh}_{x} \mathrm{As}$, $x=0.027$ shown in Fig. 1(e). Finally we mention that there is no $k_{z}$ dependence of the $\beta$ values [13].

Discussion. Our experimental results can be compared with those from theoretical calculations in the framework of DFT combined with dynamical mean-field theory (DMFT). Interestingly, non-Fermi-liquid-like self-energies have been reported [36,37] which could be fitted by sublinear power laws over a range of elevated energies. Although the detailed interpretation differs between the two references, this incoherent-metal behavior has been attributed to an interplay of Hubbard and Hund's-rule couplings in a multiband system: there is a sizable regime where carriers are strongly scattered by slowly fluctuating unquenched spins. Importantly, this source of scattering is essentially local in space, implying that it is weakly influenced by longer-range spin correlations and can thus persist over an extended part of the phase diagram. We believe that our observation of a strong, weakly doping-dependent, and approximately energy-linear singleparticle scattering rate is consistent with originating from local correlation physics as the one described by DMFT. We point out, however, that the doping dependence of the scattering rate as reported in Ref. [36], where non-Fermi-liquid behavior has been seen only for hole but not for electron doping, appears inconsistent with our results.

In the highly correlated systems inelastic scattering processes are determined by intra- (having the same orbital character) and interband (having different orbital character) transitions. The difference in the scattering rates between the hole pockets have been predicted by theoretical calculations $[22,38]$ as the scattering by intraband transitions should be larger than that by interband transitions. Figure 1(a) shows that there is a nesting between sections on the inner hole pocket having $y z$ orbital character (around point 1) and a section of the electron pocket at $M_{x}$ (around point 5) having the same orbital character. The same happens when turning the angle by $90^{\circ}$ for a nesting of sections of inner hole pocket having $x z$ orbital character with similar sections of the electron pocket at $M_{y}$. The middle hole pocket, on the other hand, shows reasonable nesting conditions only between sections having different orbital character, which explains the reduced scattering rate.

From the scattering rates we calculate the imaginary part of the self-energy function $\operatorname{Im} \Sigma$ and extrapolate the linear-in-energy-dependence to zero energy. Furthermore, we use a cutoff energy of $1.5 \mathrm{eV}$ (a value which roughly corresponds to the bandwidth of the Fe $3 d$ states) above which $\operatorname{Im} \Sigma$ is assumed to be constant. Details are described in the Supplemental Material [13]. In this way we obtain a self-energy also used in the phenomenological marginal Fermi-liquid theory [39]. The comparison with experimental data near point 1 for $\mathrm{BaFe}_{2}\left(\mathrm{As}_{1-x} \mathrm{P}_{x}\right)_{2}$ yields a coupling constant $\lambda_{M F}=1.5$ which is comparable to the values derived for cuprates [40]. Further values for $\lambda_{M F}$ are presented in the Supplemental Material [13].

Since $\operatorname{Re} \Sigma$ and $\operatorname{Im} \Sigma$ are connected by the Kramers-Kronig relation, we should also use a non-Fermi-liquid analytic expression for $\operatorname{Re} \Sigma[13,39]$. The renormalized dispersion is derived using the calculated $\operatorname{Re} \Sigma$ and the bare particle dispersion from DFT band structure calculations [41,42]. In Fig. 1(1) we show an energy distribution map of $\mathrm{BaFe}_{2}\left(\mathrm{As}_{1-x} \mathrm{P}_{x}\right)_{2}$, $x=0.24$ measured near $\Gamma$ along cut $\mathrm{I}$ using a vertical photon polarization. The dispersion could be fitted (red line) using the calculated renormalized dispersion, depending on the coupling constant $\lambda_{M F}$. We obtain a $\lambda_{M F}=1.6$ which is close to the value derived from $\operatorname{Im} \Sigma$.

In the following we calculate the renormalized dispersion close to the Fermi level as a function of the band position using $\operatorname{Re} \Sigma_{M F}$ and the parameters $\lambda_{M F}=1.5, E_{c}=1.5 \mathrm{eV}$, and an electron mass derived from DFT calculations on the inner hole pocket of $\mathrm{BaFe}_{2} \mathrm{As}_{2}$ [41]. In Fig. 2(a) we show such calculations for a bare particle hole pocket touching the chemical potential (black), for $\operatorname{Re} \Sigma$ (green), and for the renormalized band (red). Due to the fact that $\operatorname{Re} \Sigma$ scales to zero logarithmically and is very close to the bare particle dispersion, there is a strong mass renormalization $\mathrm{m}^{*} / \mathrm{m}_{0} \approx 10$ close to the Fermi level which is related to a weakly dispersive band. This mass enhancement near the Fermi level increases with increasing $\lambda_{M F}$ and increasing $m_{0}$. The mass enhancement is energy dependent and decreases at higher energy to a value $m^{*} / m_{0} \approx 2$. In Fig. 2(b) we depict an analogous calculation but with the top of the bare hole pocket shifted $50 \mathrm{meV}$ above 


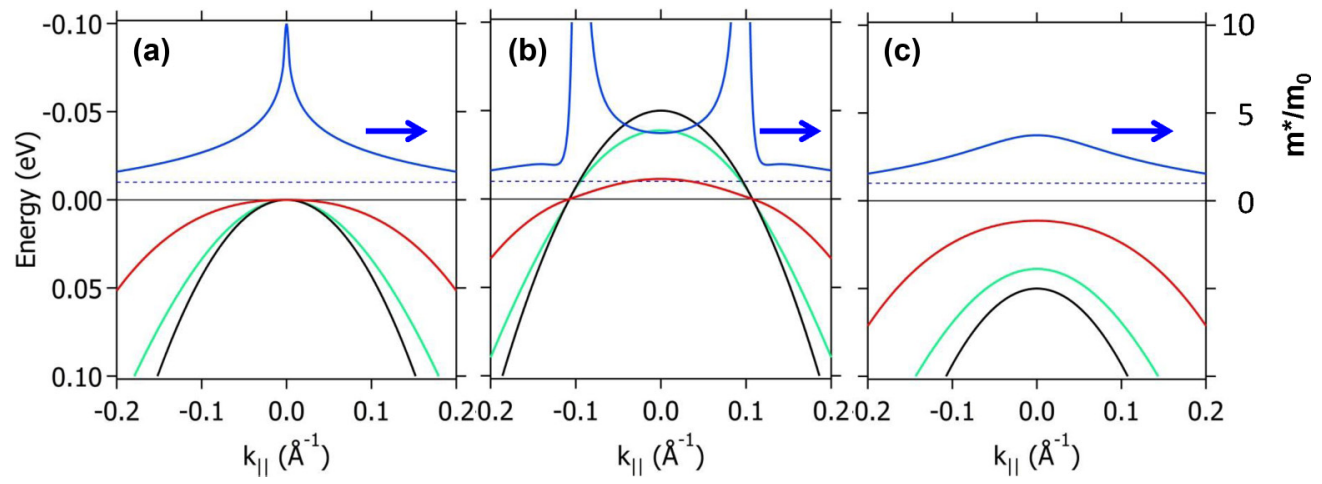

FIG. 2. (Color online) (a) Bare particle dispersion (black line) with the top of the hole pocket at the Fermi level together with calculations of $\operatorname{Re} \Sigma_{M F}$ (green line) and the renormalized dispersion (red line). In addition, we show the effective mass $m^{*} / m_{0}$ (blue line) and the $m^{*} / m_{0}=1$ line (thin dashed blue line). (b) The same as (a) but with the top of the hole pocket $50 \mathrm{meV}$ above the Fermi level. (c) The same as (a) but with the top of the hole pocket $50 \mathrm{meV}$ below the Fermi level.

the Fermi level. At the $\Gamma$ point the calculated dispersion shows a mass renormalization by a factor of 4 . On the other hand, the slope of the dispersion at the Fermi level (red line) has strongly increased. Compared to the bare particle mass $m_{0}$, below and at the Fermi level there is only a moderate mass enhancement for the occupied electronic states. We emphasize that in this case, where the top of the hole pocket is not at the Fermi level, there is no divergent mass enhancement. This means that the strong low-energy scattering rates of a moderately correlated system alone cannot describe the strange normal state properties at a specific value of the control parameter. In Fig. 2(c) we present analogous calculations for a bare particle hole pocket shifted below $50 \mathrm{meV}$ below the Fermi level. At $\Gamma$ the bare particle dispersion is renormalized by a factor $\approx 5$ and the mass enhancement is strongly reduced. Analogous calculations, in which $\operatorname{Im} \Sigma$ is extrapolated below $5 \mathrm{meV}$ by a Fermi-liquid behavior $\left(\operatorname{Im} \Sigma \propto E^{2}\right)$ yield also a large mass enhancement at the point where the hole pocket crosses the Fermi level [13].

These calculations show that the crossing of the top of the hole pocket having there a constant bare density of states typical of a two-dimensional electronic structure plays an important role for these correlated systems. It explains the divergent mass enhancement in the transport properties observed in $\mathrm{BaFe}_{2}\left(\mathrm{As}_{1-x} \mathrm{P}_{x}\right)_{2}$ at low energies near optimal doping [4] and the strange behavior of the thermal properties of $\mathrm{Ba}\left(\mathrm{Fe}_{1-x} \mathrm{Co}_{x}\right)_{2} \mathrm{As}_{2}$ [5]. Furthermore, it is interesting to compare our doping-dependent results of $\operatorname{Im} \Sigma$ with the temperature-dependent resistivity data of $\mathrm{BaFe}_{2}\left(\mathrm{As}_{1-x} \mathrm{P}_{x}\right)_{2}$ which is determined mainly by the high mobility of the charge carriers of the electron pockets [4,43]. At higher temperatures the resistivity near optimal doping is linear in temperature over a considerable range of $\mathrm{P}$ concentrations. This indicates that near optimal doping both $\operatorname{Im} \Sigma$ and the resistivity is determined by the same non-Fermi-liquid scattering rates.

There are several ARPES publications on iron-based superconductors which show clear indications of weakly dispersing bands at the Fermi level, which cannot be explained on the basis of DFT calculations. In a recent ARPES study of ferrochalcogenides an apparently nondispersive peak at $\mu$ has been detected which is not in agreement with DFT calculations [44]. Moreover, in the same system "shallow pockets" have been detected which could lead to a BCS-BE crossover in the superconducting phase [45]. These anomalous band dispersions can be well described by the scenario presented here.

Different from the iron-based superconductors, in the cuprates there is already in the bare particle band structure a peaked density of states (DOS) due to a saddle point in the band structure. There is a long-standing discussion on the importance of this van Hove singularity, which crosses the Fermi level near optimal doping [8-10] and on a breakdown of the Migdal theorem [46] which was the basis of the standard BCS theory of superconductivity. This breakdown requires the inclusion of nonadiabatic effects and the generalization of the Eliashberg equations [47] interpolating between the BCS theory and the $\mathrm{BE}$ condensation [48]. In an ARPES study on $\mathrm{YBa}_{2} \mathrm{Cu}_{3} \mathrm{O}_{7-\delta}$ an "extended" van Hove singularity has been found [49] which can be nicely explained by the scenario described above: a co-action between a highly correlated electron liquid behavior and a weakly dispersing band (the saddle point) moving through the Fermi level.

Summary. For chemically pressurized and $n$-type doped ferropnictides we observe linear-in-energy scattering rates which do not change as a function of the control parameter. In particular, we see no enhancement of the scattering rates close to the expected QCP. Furthermore, at optimal "doping" the top of the hole pockets having the largest scattering rates crosses the Fermi level. The strange normal state transport and thermal properties near optimal "doping" can be explained by a proposed scenario in which a co-action of the real part of a non-Fermi-liquid self-energy with an edge in the DOS at the Fermi level leads to a weakly dispersing band at the Fermi level and to strongly enhanced effective masses. The high effective masses imply small effective Fermi energies which could be comparable to bosonic energies mediating superconducting pairing. The possible violation of Migdal's theorem could lead to a superconducting phase which is near the BCS-BE crossover. The results can be generalized to other unconventional superconductors and possibly are a recipe for future search of new high- $T_{c}$ superconductors.

Acknowledgment. This work was financially supported by the German Research Foundation the DFG through the priority program SPP1458. 
[1] H. v. Löhneysen, A. Rosch, M. Vojta, and P. Wölfle, Rev. Mod. Phys. 79, 1015 (2007).

[2] P. Gegenwart, Q. Si, and F. Steglich, Nat. Phys. 4, 186 (2008).

[3] D. C. Johnston, Adv. Phys. 59, 803 (2010).

[4] J. G. Analytis, H.-H. Kuo, R. D. McDonald, M. Wartenbe, P. M. C. Rourke, N. E. Hussey, and I. R. Fisher, Nat. Phys. 10, 194 (2014).

[5] C. Meingast, F. Hardy, R. Heid, P. Adelmann, A. Böhmer, P. Burger, D. Ernst, R. Fromknecht, P. Schweiss, and T. Wolf, Phys. Rev. Lett. 108, 177004 (2012).

[6] J. Dai, Q. Si, J.-X. Zhu, and E. Abrahams, Proc. Natl. Acad. Sci. 106, 4118 (2009).

[7] E. Abrahams and Q. Si, J. Phys.: Condens. Matter 23, 223201 (2011).

[8] P. C. Pattnaik, C. L. Kane, D. M. Newns, and C. C. Tsuei, Phys. Rev. B 45, 5714 (1992).

[9] R. Markiewicz, J. Phys. Chem. Solids 58, 1179 (1997).

[10] G. Kastrinakis, Physica C 317-318, 497 (1999).

[11] A. Damascelli, Z. Hussain, and Z.-X. Shen, Rev. Mod. Phys. 75, 473 (2003).

[12] G. D. Mahan, Many-Particle Physics (Kluwer Academic/Plenum, New York, 2000).

[13] See Supplemental Material at http://link.aps.org/supplemental/ 10.1103/PhysRevB.92.201106 for evaluation, additional data of the scattering rates, and further calculations on the basis of a Fermi-liquid/non-Fermi liquid model.

[14] W. Malaeb, T. Yoshida, A. Fujimori, M. Kubota, K. Ono, K. Kihou, P. M. Shirage, H. Kito, A. Iyo, H. Eisaki, Y. Nakajima, T. Tamegai, and R. Arita, J. Phys. Soc. Jpn. 78, 123706 (2009).

[15] C. He, Y. Zhang, B. P. Xie, X. F. Wang, L. X. Yang, B. Zhou, F. Chen, M. Arita, K. Shimada, H. Namatame, M. Taniguchi, X. H. Chen, J. P. Hu, and D. L. Feng, Phys. Rev. Lett. 105, 117002 (2010).

[16] S. Thirupathaiah, S. de Jong, R. Ovsyannikov, H. A. Dürr, A. Varykhalov, R. Follath, Y. Huang, R. Huisman, M. S. Golden, Y.-Z. Zhang, H. O. Jeschke, R. Valenti, A. Erb, A. Gloskovskii, and J. Fink, Phys. Rev. B 81, 104512 (2010).

[17] S. Thirupathaiah, E. D. L. Rienks, H. S. Jeevan, R. Ovsyannikov, E. Slooten, J. Kaas, E. van Heumen, S. de Jong, H. A. Dürr, K. Siemensmeyer, R. Follath, P. Gegenwart, M. S. Golden, and J. Fink, Phys. Rev. B 84, 014531 (2011).

[18] Z.-H. Liu, P. Richard, K. Nakayama, G.-F. Chen, S. Dong, J.-B. He, D.-M. Wang, T.-L. Xia, K. Umezawa, T. Kawahara, S. Souma, T. Sato, T. Takahashi, T. Qian, Y. Huang, N. Xu, Y. Shi, H. Ding, and S.-C. Wang, Phys. Rev. B 84, 064519 (2011).

[19] T. Yoshida, I. Nishi, S. Ideta, A. Fujimori, M. Kubota, K. Ono, S. Kasahara, T. Shibauchi, T. Terashima, Y. Matsuda, H. Ikeda, and R. Arita, Phys. Rev. Lett. 106, 117001 (2011).

[20] M. Yi, D. H. Lu, R. G. Moore, K. Kihou, C.-H. Lee, A. Iyo, H. Eisaki, T. Yoshida, A. Fujimori, and Z.-X. Shen, New J. Phys. 14, 073019 (2012).

[21] Z. R. Ye, Y. Zhang, F. Chen, M. Xu, J. Jiang, X. H. Niu, C. H. P. Wen, L. Y. Xing, X. C. Wang, C. Q. Jin, B. P. Xie, and D. L. Feng, Phys. Rev. X 4, 031041 (2014).

[22] A. F. Kemper, M. M. Korshunov, T. P. Devereaux, J. N. Fry, H.-P. Cheng, and P. J. Hirschfeld, Phys. Rev. B 83, 184516 (2011).
[23] J. Bardeen, L. N. Cooper, and J. R. Schrieffer, Phys. Rev. 108, 1175 (1957).

[24] S. N. Bose, Z. Phys. 26, 178 (1924).

[25] H. S. Jeevan, D. Kasinathan, H. Rosner, and P. Gegenwart, Phys. Rev. B 83, 054511 (2011).

[26] F. Steckel, M. Roslova, R. Beck, I. Morozov, S. Aswartham, D. Evtushinsky, C. G. F. Blum, M. Abdel-Hafiez, D. Bombor, J. Maletz, S. Borisenko, A. V. Shevelkov, A. U. B. Wolter, C. Hess, S. Wurmehl, and B. Büchner, Phys. Rev. B 91, 184516 (2015).

[27] E. van Heumen, J. Vuorinen, K. Koepernik, F. Massee, Y. Huang, M. Shi, J. Klei, J. Goedkoop, M. Lindroos, J. van den Brink, and M. S. Golden, Phys. Rev. Lett. 106, 027002 (2011).

[28] A. Lankau, K. Koepernik, S. Borisenko, V. Zabolotnyy, B. Büchner, J. van den Brink, and H. Eschrig, Phys. Rev. B 82, 184518 (2010).

[29] V. Brouet, M. Fuglsang Jensen, A. Nicolaou, A. TalebIbrahimi, P. Le Fevre, F. Bertran, A. Forget, and D. Colson, arXiv:1105.5604.

[30] E. D. L. Rienks, T. Wolf, K. Koepernik, I. Avigo, P. Hlawenka, C. Lupulescu, T. Arion, F. Roth, W. Eberhardt, U. Bovensiepen, and J. Fink, Europhys. Lett. 103, 47004 (2013).

[31] M. Yi, D. Lu, J.-H. Chu, J. G. Analytis, A. P. Sorini, A. F. Kemper, B. Moritz, S.-K. Mo, R. G. Moore, M. Hashimoto, W.-S. Lee, Z. Hussain, T. P. Devereaux, I. R. Fisher, and Z.-X. Shen, Proc. Natl. Acad. Sci. USA 108, 6878 (2011).

[32] I. R. Fisher, L. Degiorgi, and Z. X. Shen, Rep. Prog. Phys. 74 124506 (2011).

[33] V. Brouet, M. Marsi, B. Mansart, A. Nicolaou, A. TalebIbrahimi, P. Le Fevre, F. Bertran, F. Rullier-Albenque, A. Forget, and D. Colson, Phys. Rev. B 80, 165115 (2009).

[34] C. Liu, T. Kondo, R. M. Fernandes, A. D. Palczewski, E. D. Mun, N. Ni, A. N. Thaler, A. Bostwick, E. Rotenberg, J. Schmalian, S. L. Bud'ko, P. C. Canfield, and A. Kaminski, Nat. Phys. 6, 419 (2010).

[35] S. Nandi, W. T. Jin, Y. Xiao, Y. Su, S. Price, W. Schmidt, K. Schmalzl, T. Chatterji, H. S. Jeevan, P. Gegenwart, and T. Brückel, Phys. Rev. B 90, 094407 (2014).

[36] P. Werner, M. Casula, T. Miyake, F. Aryasetiawan, A. J. Millis, and S. Biermann, Nat. Phys. 8, 331 (2012).

[37] Z. P. Yin, K. Haule, and G. Kotliar, Phys. Rev. B 86, 195141 (2012).

[38] S. Graser, T. A. Maier, P. J. Hirschfeld, and D. J. Scalapino, New J. Phys. 11, 025016 (2009).

[39] C. Varma, Z. Nussinov, and W. van Saarloos, Phys. Rep. 361, 267 (2002).

[40] T. Valla, A. V. Fedorov, P. D. Johnson, and S. L. Hulbert, Phys. Rev. Lett. 83, 2085 (1999).

[41] D. J. Singh, Phys. Rev. B 78, 094511 (2008).

[42] S. Deng, J. Köhler, and A. Simon, Phys. Rev. B 80, 214508 (2009).

[43] S. Kasahara, T. Shibauchi, K. Hashimoto, K. Ikada, S. Tonegawa, R. Okazaki, H. Shishido, H. Ikeda, H. Takeya, K. Hirata, T. Terashima, and Y. Matsuda, Phys. Rev. B 81, 184519 (2010).

[44] P. Starowicz, H. Schwab, J. Goraus, P. Zajdel, F. Forster, J. R. Rak, M. A. Green, I. Vobornik, and F. Reinert, J. Phys.: Condens. Matter 25, 195701 (2013). 
[45] Y. Lubashevsky, E. Lahoud, K. Chashka, D. Podolsky, and A. Kanigel, Nat. Phys. 8, 309 (2012).

[46] A. B. Migdal, Sov. Phys. JETP 34, 996 (1958).

[47] C. Grimaldi, L. Pietronero, and S. Strässler, Phys. Rev. Lett. 75, 1158 (1995).
[48] N. Andrenacci, A. Perali, P. Pieri, and G. C. Strinati, Phys. Rev. B 60, 12410 (1999).

[49] K. Gofron, J. C. Campuzano, A. A. Abrikosov, M. Lindroos, A. Bansil, H. Ding, D. Koelling, and B. Dabrowski, Phys. Rev. Lett. 73, 3302 (1994). 